\title{
Exploiting Diversity in Future Generation Satellite Systems with Optical Feeder Links
}

\author{
A. Mengali, F. Kayhan, B. Shankar and B. Ottersten \\ Interdisciplinary Centre for Security, Reliability and Trust (SnT), University of Luxembourg, Luxembourg
}

\begin{abstract}
In this paper we exploit the transmitter micro diversity in the satellite communication systems with optical feeder links. Both analogue and digital transparent systems are studied. We consider up to four statistically independent transmitted optical beams with perfect or partial phase knowledge of the beams at the receiver. End-to-end simulation results for frame error rates are presented for a complete system including uplink, satellite payload and downlink. We compare our results with those of the DVB-S2 standard without the optical feeder link. Our results provide a complete view of gains and losses which can be obtained by micro diversity in both analogue and digital transparent satellite systems with optical feeder link. Given the system parameters, we also provide a detailed study of the transmitted power needed to achieve a target frame error rate and discuss the achieved bit rates in for each system scenario.
\end{abstract}

\section{Introduction}

The steady growth in data traffic due to new services and the increasing number of communicating devices mandates a constant up-grading of all communication networks including satellite systems. Leveraging on wide coverage, satellite communications are evolving from traditional TV broadcasting systems into systems that offer broadband services, machine-to-machine connectivity, mobility support to maritime and aeronautical terminals, etc. ${ }^{1}$ In 2020, the required capacity for satellite broadband services in Europe alone is foreseen to be about 5.3 Terabit/s. ${ }^{2}$

To meet the enhanced data requirements imposed by the user link, it is imperative that the feeder link has the necessary wherewithal to uplink the aggregate traffic. This has driven recent research in satellite communications into the study of provisioning high bandwidth feeder links. Since the limited spectrum available in $\mathrm{Ku}-\mathrm{Ka}$ bands may not be sufficient to ensure Terabit/s communications by means of efficient frequency reuse alone, most feeder link studies focus on moving to higher frequency bands (Radio Frequency $(\mathrm{RF})$ bands like Q/V $(40 / 50 \mathrm{GHz}),{ }^{3-6} \mathrm{~W}(70 / 80 \mathrm{GHz})^{7}$ or even Optical. ${ }^{8-10}$ Free Space Optics (FSO) communications represent an interesting alternative due to the following advantages over $\mathrm{RF}^{8}{ }^{8}$ a) 100 to 1000 times higher available bandwidth, b) lower power requirements and smaller antennas, c) no spectrum regulatory constraints d) High resistance to interception due to the extremely directive beams. Optical frequencies, on the other hand, are heavily impaired by many atmospheric phenomena: blocking of the Line of Sight (LOS) predominantly by clouds and fog, (and to a lesser extent, by rains) as well as fading introduced by air turbulence even in cloud-free line of sight (CFLOS) conditions. Despite their apparent severe drawbacks, FSO communications are worthy of being investigated for two main reasons. First, the impairments induced by clouds and turbulence depend on the transmission frequency, and some of the drawbacks of FSO also become relevant, although to a lesser extent, in higher RF bands. ${ }^{4}$ Second, their bandwidth availability makes them attractive for future generation systems, ${ }^{2}$ where it may be challenging to achieve extremely high throughputs with the limited RF usable spectrum.

The current asymmetry in data communications between the forward and return links as well as the challenges imposed by optical frequencies (limited coverage, exposure to radiation) encourage the use of hybrid systems, where user links continue to exist in RF (possibly including frequencies formerly allocated to feeder link) and feeder link is provisioned in optical frequencies.

One way to avoid cloud blockage, is to utilize a network of multiple Optical Gateway Station (OGS) that are geographically dispersed, at least as far apart as the meteorological correlation length of large cloud 
cover structures, such that there is a high probability of CFLOS from a ground station at any given point in time. This is usually referred to transmitter macro diversity. One of the main drawbacks of this approach is the cost of having several ground stations.

Beside this diversity on a macroscopical scale, the use of multiple co-located transmitting/receiving elements (micro-diversity) has been among the most effective ways of mitigating the effect of atmospheric turbulence in optical wireless communications. ${ }^{11}$ Many works have been focused on exploiting the diversity gain achievable from Multiple Input Multiple Output (MIMO) optical setups. Among the spatial multiplexing methods that have received particular interest, we can find: Optical Spatial Modulation (OSM) $)^{11,12}$ Repetition Coding (RC) ${ }^{8,13,14}$ and Optical Space Time Codes (OSTC). ${ }^{15-19}$

In this paper we will focus on the exploitation of micro diversity to achieve flexibility and efficiency in the deployment of a hybrid optical/RF communication system. To ensure system longevity and adaptation to possible changes in the air interface scheme used (e.g. DVB-S2X), we will limit our study to transparent payload architectures, either fully analogue or partially digital. ${ }^{20}$ In case of Analogue Transparent Scheme (ATS) payload, we convert the analogue RF signal into the optical domain and then switch back again to electrical on-board the satellite. For the Digital Transparent Scheme (DTS), the RF signal is digitized, through an analog-to-digital converter (ADC), prior to the optical transmission and reconstructed from its digital form, through a digital-to-analog converter (DAC), at the output of the optical receiver on-board the satellite. This second approach provides additional flexibility and robustness at the expense of added complexity.

One of our goals in this paper is to show that the performance of the micro diversity depends strongly on the knowledge of the phases of each received beam. When perfect knowledge of the beam's phases are available, the loss from the DVB-S2 is usually very small. On the other hand, assuming a random phase, the loss can increase to several dBs. We also study the effect of having only partial knowledge of the beams' phases at the receiver.

In order to have a realistic view, our end-to-end simulation chain includes all the essential blocks of transmission, i.e., optical uplink channel, satellite payload and downlink channel. We measure the Frame Error Rate (FER) at the user terminal and compare the performances with the DVB-S2 standard. Several interesting observations can be made from our simulations. First of all, the micro diversity is beneficial only if some knowledge regarding the phases of each beam is assumed. The results also show that the DTS needs lower power per chain to achieve the same performance as the ATS. Moreover, our simulations indicate that the number of transmitted beams can be reduced to only two when the scintillation index is small.

The rest of this paper is organized as follows. In section II we provide a short description of the DTS and ATS system models. We also present in some details the blocks implemented in our simulator. The simulation parameters and the comparison between the transmitted power and bit rate for both system models are presented in section III. In section IV, we present the simulation results for both ATS and DTS. Finally, we conclude the paper in section V.

\section{Notations and system model}

In this section we provide a short description of DTS and ATS. We also present the end-to-end simulation chain and explain briefly each implemented block.

\section{A. System model}

In Figure 1 we show the system model considered in this paper. The transmitted data is first processed using the DVB-S2 standard including baseband filtering. The filtered samples are then converted to the optical domain, using either ATS or DTS, before being transmitted through the optical channel. At the satellite, the optical signal is reconverted into (RF) and is sent through the downlink channel after being filtered and amplified on board the satellite.

\section{B. Analogue and digital transparency in satellite systems with optical feeder links}

The "transparency" of a satellite, meaning its ability to act as a relay without the knowledge of the airinterface used for the transmission, is often a a key requirement for satellite operators. This permits a much higher flexibility in the use of the satellite but implies that no processing of the user streams (demodulation, decoding, etc..) can be done on-board. This paper focuses on transparent payloads to assess the feasibility of 


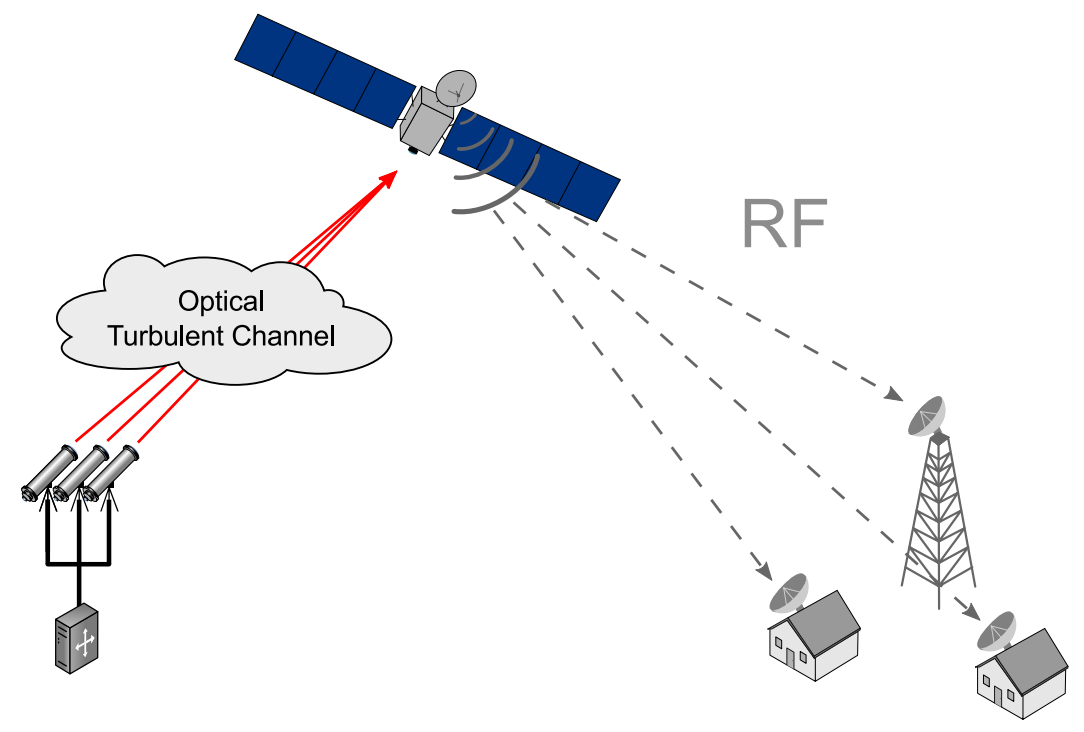

Figure 1. System model

employing feeder links without completely redesigning the space segment. This requirement directly impacts the feeder link design as the RF user signals must be treated as analogue. Two transparency schemes are usually considered in the literature. In the following, we briefly describe each one.

\section{Analogue transparent system}

In the ATS, at the OGS, the users' signals from the DVB-S2 chain ${ }^{21}$ are aggregated and directly modulated onto the optical carrier using intensity modulation. On-board the satellite, the signal is first optically preamplified by means of a Low Noise Optical Amplifier (LNOA), and then it is converted by a square law opto-electrical detector to recover the original DVB-S2 signals. This transmission scheme usually suffers from extreme turbulence-fading and only physical layer mitigation techniques can be applied to counteract this effect. Such techniques include transmitter diversity at the OGS and Automatic Gain Control (AGC) functionalities on-board the satellite. The optical modulation used in such systems is usually Subcarrier Intensity Modulation (SIM), which has been extensively analyzed in literature. ${ }^{22-25}$ In SIM, since the output light amplitude can only be positive, an electrical bias needs to be added to the aggregate signal to ensure its non-negativity. The main advantage of this scheme is the simple implementation and low cost, the disadvantage is that a significant portion of the transmit power is wasted on the bias.

\section{Digital transparent scheme}

In the DTS, the digitized samples of the aggregate signal are transmitted over the optical feeder link, after an ADC on the ground. Several modulation schemes are proposed in the literature to transmit the digitized signal. In this paper we focus only on the simplest modulation scheme, namely On-Off Keying (OOK). Therefore, this scheme also requires a DAC on-board of the satellite. In exchange for this more complex design one can maintain flexibility to new optical modulation and coding techniques. On the other hand, this approach offers relatively low spectrum efficiency in the optical channel, because oversampling and quantization are required. The bandwidth requirements are therefore much higher than the analogue transparent approach. A detailed discussion on this problem is provided in Section III.

\section{Simulation chain}

We simulate the FER of both ATSs and DTSs as a function of downlink Signal-to-Noise Ratio (SNR). The complete chain including the ground station, optical uplink channel, satellite payload, downlink channel and 
user terminal is simulated. In Figures 2(a) and 2(b) we present the simulation blocks for the analogue and digital scheme respectively. In the following we analyze both chains in more details.

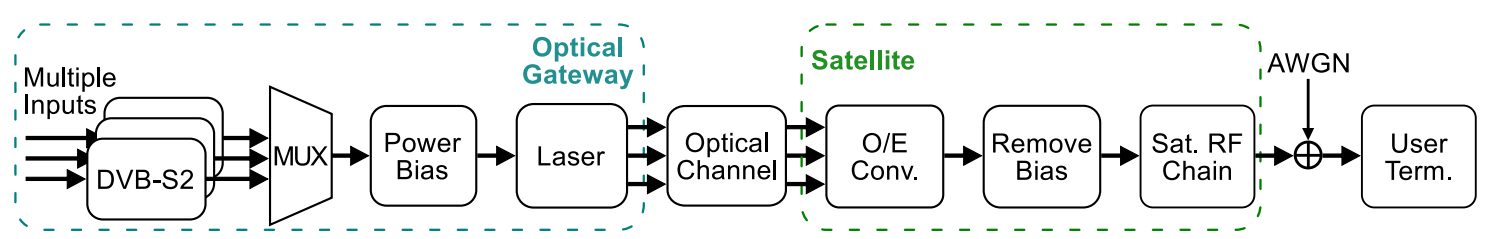

(a) Analog Transparent

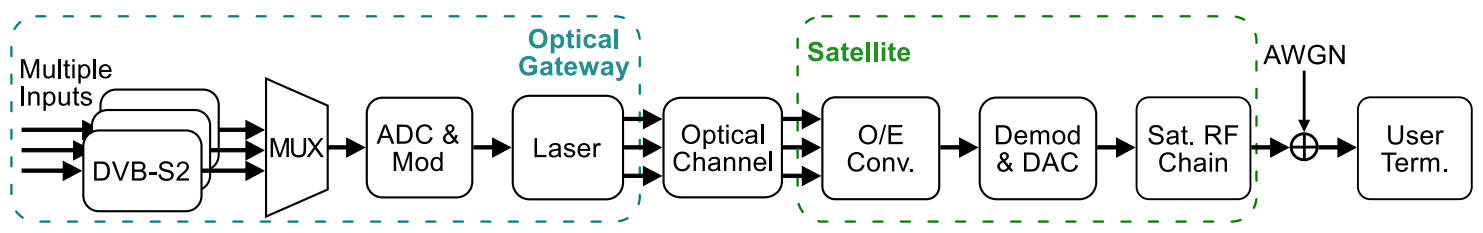

(b) Digital Transparent

Figure 2. Simulation block diagrams.

\section{Optical gateway}

In both architectures, multiple data streams are first processed to the DVB-S2 format and multiplexed together in frequency. In the analogue case, as explained in subsection B, this aggregate signal is modulated (using SIM) onto one or multiple lasers, depending on the required micro-diversity. In the digital case, the samples of the aggregate signal are quantized into bits and mapped into OOK pulses, used to drive the lasers.

\section{Optical channel}

The effect of the optical turbulent channel on the signal amplitude is usually modeled as a multiplicative noise. $^{26}$ The severity of the turbulence is identified by the Scintillation Index (SI), defined as the variance of the received signal irradiance $(I)$ divided by its squared mean: ${ }^{26}$

$$
\sigma_{I}^{2}=\frac{\left\langle[I-\langle I\rangle]^{2}\right\rangle}{\langle I\rangle^{2}}
$$

where $\langle\cdot\rangle$ represents the expectation operator. In this work, we assume a lognormal distribution of the signal irradiance as previously done in other works. ${ }^{27-30}$ This distribution is usually used for weak to moderate turbulence, i.e. $\sigma_{I}^{2}<1$, and has been shown to match some optical satellite link measurements. For an example see the measurements conducted by the European Space Agency. ${ }^{31}$ In our simulations, we assume that the same average power level is kept during a full DVB-S2 frame duration. This is because the coherence time of the optical channel usually falls in the order of milliseconds ${ }^{32}$ and can thus be assumed longer than the duration of a single DVB-S2 frame.

\section{Satellite: optical to electrical conversion}

When light arrives at the satellite receiver, it has to be converted to an electrical signal. This process follows various steps as seen in Figure 3(a). In our simulations, we consider only one receiving antenna on-board the satellite to limit the payload mass and complexity. The light from multiple transmitting beams is combined on the single receiving aperture. At this point, due to Free Space Loss (FSL), the optical signal power is very low and is thus amplified using an Erbium-Doped Fiber Amplifier (EDFA). Inside the optical amplifier, when the temperature is above $0^{\circ} \mathrm{K}$, an interfering optical wave is generated by the spontaneous emission of photons. ${ }^{33}$ This additional optical signal is also amplified while passing through the EDFA and translates into a significant noise term called Amplified Stimulated Emission (ASE) Noise. An optical filter is implemented in order to reduce the effect of the ASE noise. As a consequence of the ASE noise some beat noise terms are produced during the frequency down-conversion performed by the PIN photodiode. On top 
of the beat noise, other noises such as dark current, shot noise and thermal noise also affect the electrical signal. The effects of these noises are reduced by another filter (electr. filter block in Figure 3(a)) before the signal is passed to the electrical pre-amplifier.

\section{Satellite: radio frequency chain}

In the analogue case, after the opto-electrical conversion block, the signal is already in it's baseband analog form with a power bias. Once this bias is removed, the signal can be fed to the RF satellite chain block expanded in Figure 3(b). First a frequency upconversion is performed to bring the analog signal to the desired working frequency of the Input Multiplexing (IMUX) filters. These split the aggregate signal into separate subchannels that are then amplified using a High Power Amplifier (HPA) and fed to the Output Multiplexing (OMUX) filter to be separated into the desired downlink beams. Before the amplification, an ideal (AGC) is assumed to bring the signal to the desired power level. The only difference in the DTS is that at the output of the opto-electrical conversion block we do not have the signal in it's analogue form. For this reason, the electrical signal is first fed to a demodulator and then to a DAC which reconstructs the analogue samples from the demodulated bits. It is important to point out that despite having a demodulation process here, it does not interact with the user data but only operates on the optical carrier used to encapsulate such data. Therefore, the transparency nature of the chain is preserved. The rest of the chain is same as the ATS.

\section{User terminal}

The user terminal chain is composed of a Least Mean Squares (LMS) Linear Equalizer with 4 taps and demodulation and decoding processes of the DVB-S2 standard.

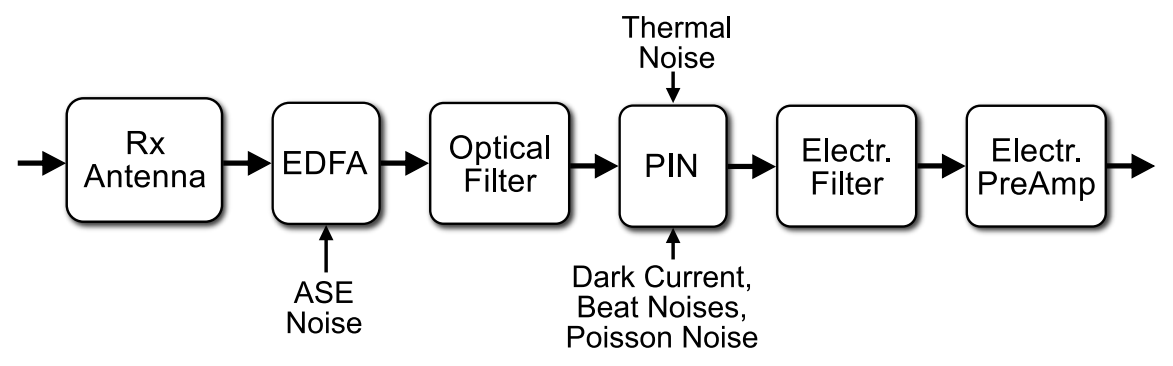

(a) Optical to Electrical Conversion

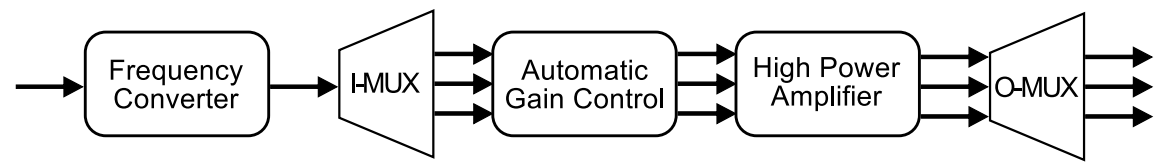

(b) Satellite Radio Frequency Chain

Figure 3. Detailed view of satellite and optical to electrical conversion blocks.

\section{Simulation parameters and discussion on needed transmitted power}

\section{A. Simulation parameters}

In all simulations the MODCOD employing the QPSK constellation and the LDCP code of rate $3 / 4$ is used. The ADC and DAC have $Q=6$ Quantization bits per dimension and an oversampling rate of $O S R=4$ is used. On the satellite side, the IMUX filter, OMUX filter and the non-linear HPA are the ones specified in the DVB-S2 standard. ${ }^{34}$ We always assume the combined effect of the electrical preamplifier and theAGC, to result in a power level at the output of the IMUX which is $2 \mathrm{~dB}$ lower than the amplifier saturation power $(\mathrm{IBO}=2 \mathrm{~dB})$. The other parameters used in the simulations are given in table $1 . \lambda$ represents the wavelength of optical system. $G_{T}$ and $G_{R}$ represent the antenna gains of the transmitter and receiver optical telescopes while $L_{\text {sys }}$ is the loss of the system due to beam wander and implementation losses. We assume the satellite to operate in a GEO orbit. The formulation for computing the various noise values presented 
in subsection $\mathrm{C}$ are taken from. ${ }^{33}$ In the following we summarize these equations. Given an incident useful signal optical power of $P_{S} \mathrm{~W}$ at the satellite telescope and considering an EDFA optical pre-amplifier with optical gain $G$, the signal electrical current at the output of the PIN photodiode can be expressed as:

$$
I_{\mathrm{S}}=R \cdot G P_{\mathrm{S}}
$$

with $R \leq 1$ being the responsivity of the PIN diode.

We denote by $P_{A S E}$ the equivalent ASE noise power of the EDFA before amplification. Then the ASE noise Direct Current (DC) component can be formulated as:

$$
I_{\mathrm{ASE}}=R \cdot G P_{\mathrm{ASE}}
$$

Shot noise is present in all currents generated by the photo-detection process, including the dark current. Considering a generic current $I$, the shot noise power relative to this current can be expressed as:

$$
\mathrm{r}_{\mathrm{sh}}^{2}=2 q I B_{\mathrm{e}}
$$

where $B_{\mathrm{e}}$ is the bandwidth of the electrical filter after the PIN. On the other hand, the beat noise power can be expressed as follows:

$$
\begin{aligned}
& i_{s i g-s p}^{2}=4 I_{\mathrm{S}} \cdot I_{\mathrm{ASE}} \cdot \frac{B_{\mathrm{e}}}{B_{\mathrm{o}}} \\
& i_{s p-s p}^{2}=I_{\mathrm{ASE}}^{2}\left(2-\frac{B_{\mathrm{e}}}{B_{\mathrm{o}}}\right) \frac{B_{\mathrm{e}}}{B_{\mathrm{o}}}
\end{aligned}
$$

where (5) represents the term relative to the product between the useful signal and the ASE noise and (6) represents the term relative to the product between the ASE noise with itself. Finally, the thermal noise in optical systems is usually defined by means of the electrical noise current spectral density $i_{n}$ as

$$
i_{\mathrm{th}}^{2}=i_{\mathrm{n}}^{2} B_{\mathrm{e}}
$$

We define as Optical Signal-to-Noise Ratio (OSNR) the ratio between the useful signal power and the total noise power introduced by the opto-electrical chain. With the aid of the previous equations, one can express OSNR in the digital case as:

$$
\mathrm{OSNR}_{\mathrm{d}}=\frac{I_{\mathrm{S}}^{2}}{i_{s i g-s p}^{2}+i_{s p-s p}^{2}+2 q\left(I_{\mathrm{S}}+I_{\mathrm{ASE}}+I_{\mathrm{d}}\right) B_{\mathrm{e}}+i_{\mathrm{th}}^{2}}
$$

In the analog case, the noise power will remain the same, but the useful signal power is lower due to the power bias introduced at the transmitter.

Let $P_{x}$ denotes the power of the useful transmitted signal, $I_{\text {bias }}$ denotes the amplitude of the bias and $P_{T}$ the total transmitted power including both the useful signal and the bias. One can easily compute the useful power ratio $\beta$ as:

$$
\beta=\frac{P_{x}}{P_{T}}=\frac{P_{x}}{P_{x}+I_{\text {bias }}^{2}} .
$$

In our simulations, we choose $I_{\text {bias }}$ as the maximum value of the signal at the output of the transmitter BB filter. For the particular modulation chosen, this leads to a value of $\beta=0.222$. The OSNR in the analogue case is then:

$$
\operatorname{OSNR}_{\mathrm{a}}=\frac{\left(\beta I_{\mathrm{S}}\right)^{2}}{i_{s i g-s p}^{2}+i_{s p-s p}^{2}+2 q\left(I_{\mathrm{S}}+I_{\mathrm{ASE}}+I_{\mathrm{d}}\right) B_{\mathrm{e}}+i_{\mathrm{th}}^{2}}
$$

\section{B. Discussion on needed transmitted power for Terabits/s in analogue and digital transparent systems}

The transparency requirement imposed to ensure adaptation of the satellite payload to changes in the air interface, causes a big bandwidth expansion of the DTS as opposed to the ATS. This is due to the necessity of quantizing the RF samples to permit using optical digital modulation schemes. 


\begin{tabular}{|l||l|l|l|l|l|l|}
\hline Parameter & $\lambda$ & $\mathrm{G}_{\mathrm{T}}$ & $\mathrm{G}_{\mathrm{R}}$ & $\mathrm{L}_{\mathrm{FSL}}$ & $\mathrm{L}_{\text {sys }}$ & $\mathrm{R}$ \\
\hline Value & $1550 \mathrm{~nm}$ & $110 \mathrm{~dB}$ & $110 \mathrm{~dB}$ & $-290.21 \mathrm{~dB}$ & $-15 \mathrm{~dB}$ & 0.5 \\
\hline \hline Parameter & $\mathrm{B}_{\mathrm{e}}$ & $\mathrm{B}_{\mathrm{o}}$ & $\mathrm{P}_{\mathrm{ASE}}$ & $\mathrm{G}$ & $\mathrm{I}_{\mathrm{d}}$ & $\mathrm{i}_{\mathrm{n}}$ \\
\hline Value & $40 \mathrm{GHz}$ & $40 \mathrm{GHz}$ & $5 \cdot 10^{-9}$ & $40 \mathrm{~dB}$ & $10^{-10} \mathrm{~A}$ & $10^{-11} \mathrm{~A} / \sqrt{\mathrm{Hz}}$ \\
\hline
\end{tabular}

Table 1. Parameters used for the simulations

Assuming to keep the same information rate between the different transmission schemes, during each symbol duration the number of bits to be transmitted optically depends on both the oversampling rate (OSR) and the quantization levels $(\mathrm{Q})$ in the ADC. This translates to a required bandwidth which is $(\mathrm{OSR} \cdot 2 \cdot \mathrm{Q})$ larger. The factor 2 is due to the complex nature of the base-band representation of the RF signal which implies 2 separate quantizations.

Even though the bandwidth availability in optical frequencies is not a problem, the maximal baud rate per chain is limited by the existing technology. On the other hand, regardless of the specific per-chain rate, the DTS always requires a higher number of optical chains.

With the parameters assumed in the our simulations ( $\mathrm{OSR}=4, \mathrm{Q}=6$ ), the transmission with OOK would require a bandwidth which is 48 times larger. Given that the total transmitted power at the gateway is often the main constraint, we decided to compare performances of the two schemes under the same total power constraint. In this paper, we fix the transmit power of the DTS to $P_{T}=50 \mathrm{~W}$. That implies that the transmit power for ATS should be fixed to $P_{T}=2400 \mathrm{~W}$ for a fair total power comparison.

We compare the performance of the DTS and ATS using these transmit power values in Figures 4(a) and 4(b) for $\sigma_{I}^{2}=0.05$ and $\sigma_{I}^{2}=0.4$ respectively, corresponding to weak and moderate turbulence scenarios. The performance of the ATS with $P_{T}=4800 \mathrm{~W}$ and $P_{T}=1200 \mathrm{~W}$ are also added in the same figure to have a broader view of the system performance. For $\sigma_{I}^{2}=0.05$, from the Figure 4 (a) we can see that with a

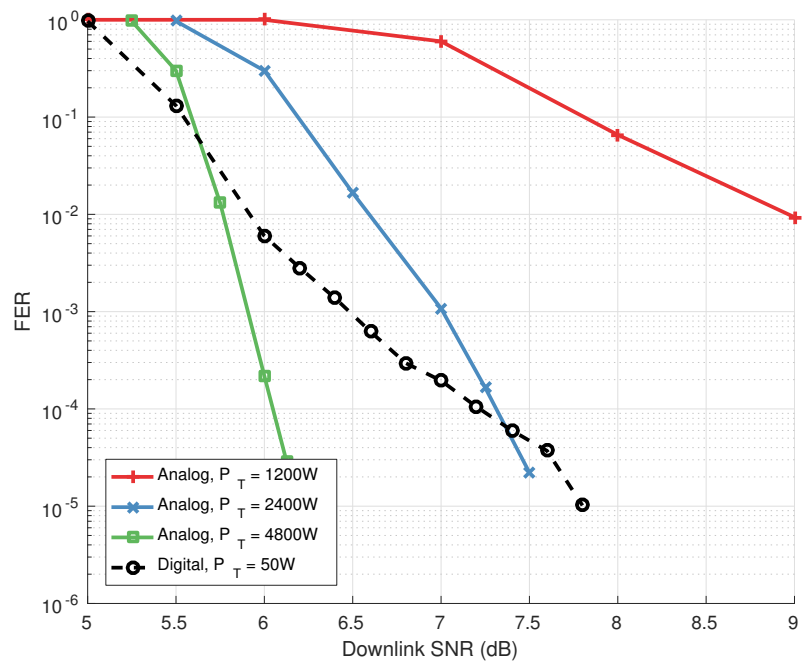

(a) $\sigma_{I}^{2}=0.05$

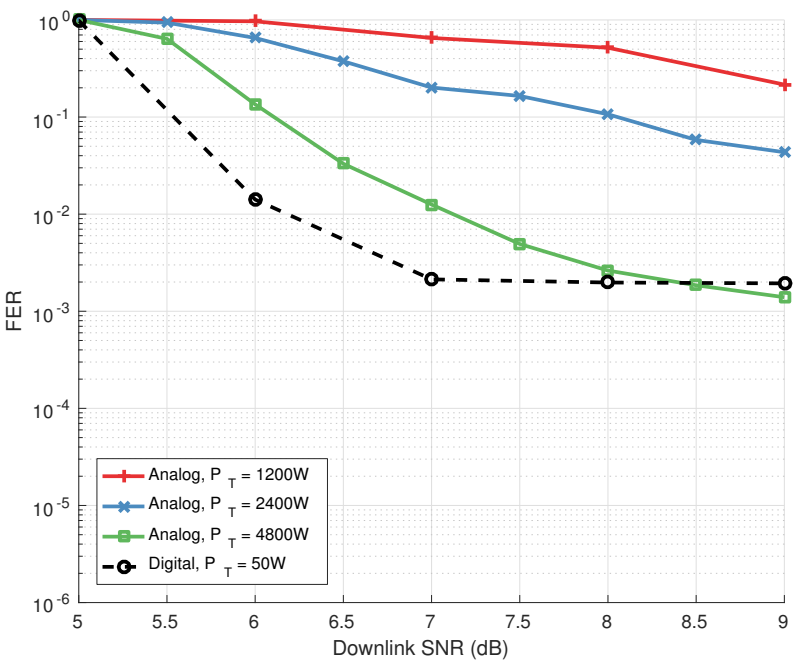

(b) $\sigma_{I}^{2}=0.4$

Figure 4. Power Comparison between analogue and digital schemes.

transmit power of $P_{T}=2400 \mathrm{~W}$ the ATS performance is near to that of the DTS at the target FER of $10^{-5}$.

\section{Micro diversity}

We considered up to 4 separated transmitters in our micro-diversity scheme. To ensure fairness in the comparison, we impose the same total transmitted power between different diversity scenarios. As the spatial coherence length of the satellite optical channel is in the order of centimeters, ${ }^{32}$ we assumed the different transmitted beams to experience independent realization of the turbulence channel. We used Repetition 
Coding (RC) as micro-diversity scheme. RC has been shown to perform well for intensity modulated optical signals. ${ }^{13}$ One aspect which has not been analyzed $i^{13}$ is the impact of random phase on the separate beams combined at the receiver aperture. While power combining provides substantial gains when the signals are coherently added, the random phase on the optical beams may lead to destructive interference during the combination. To consider the possibility of exploiting a partial knowledge of the optical phases, we simulated diversity assuming different level of phase alignment in the 4 beams scenario. From completely coherent combination (indicated by "pha $=0$ " in the rest of the paper), to completely random phase for each beam $(\mathrm{pha}=100)$. The intermediate values corresponds to random phase in the range $[0, \pi / 2](\mathrm{pha}=$ $25)$ and $[0, \pi](\mathrm{pha}=50)$.

\section{A. Digital transparent}

First we focus on the digital transparent scheme. We consider the same two SI values used in section III. The simulation results for $\sigma_{I}^{2}=0.05$ are presented in Figure 5(a). In the same figure, we also report the performance of the DVB-S2 without optical feeder link ("DVB-S2, $\mathrm{m}=2, \mathrm{r}=34$ ") for comparison. As it can be observed, the loss without exploiting the diversity $(\mathrm{ntx}=1$, pha $=0)$ is more than $2 \mathrm{dBs}$. This loss can be completely compensated using 2 beams if the perfect knowledge of phases is available at the receiver. In case of partial knowledge with pha $=25$, four beams are needed in order to avoid any loss.

Similar observation can be made $\sigma_{I}^{2}=0.4$ in Figure 5(b). The main difference here is that even with perfect knowledge of the phases, two beams are not sufficient. Also notice that no optical coding has been used in our simulations to achieve these results and therefore, only the optical demodulator is needed on board the satellite in our scheme.

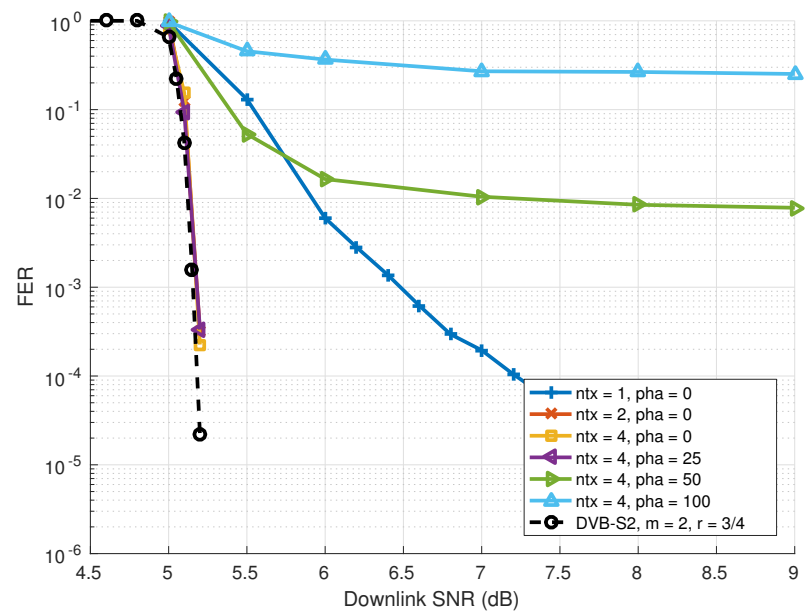

(a) $\sigma_{I}^{2}=0.05$

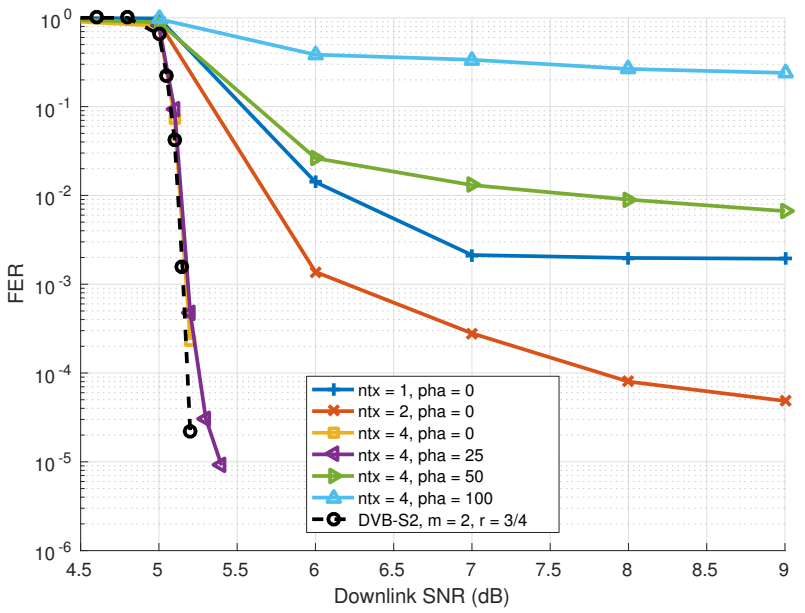

(b) $\sigma_{I}^{2}=0.4$

Figure 5. Micro diversity in digital transparent systems with $P_{T}=50 \mathrm{~W}$.

\section{B. Analogue transparent}

In this part we focus on the analogue transparent scheme. In line with the discussion of section III, we choose a transmit power $P_{T}=2400 \mathrm{~W}$. As before, two SI values are considered: $\sigma_{I}^{2}=0.05$ and $\sigma_{I}^{2}=0.4$. The general behavior of ATS is similar to that of the DTS. However, in almost all cases, the performance is degraded by $0.2 \mathrm{~dB}$ to $0.8 \mathrm{~dB}$ depending on diversity scheme. In particular, notice that for $\sigma_{I}^{2}=0.05$, by using 2 beams and having perfect phase knowledge at receiver, the loss from the DVB-S2X chain (without optical feeder link) is only $0.8 \mathrm{~dB}$ at target FER of $10^{-5}$. The simplicity of the system implementation may indeed justify this small loss with respect to the DTS case. 


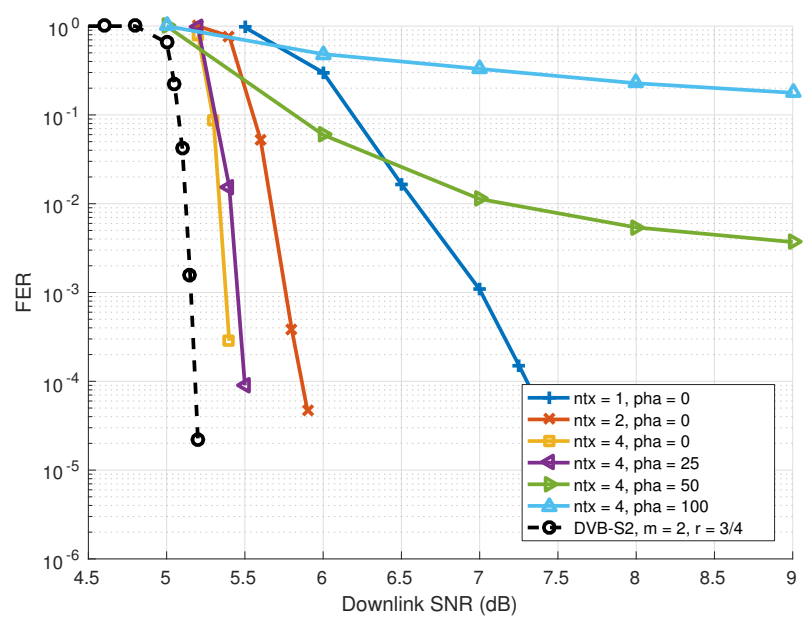

(a) $\sigma_{I}^{2}=0.05$

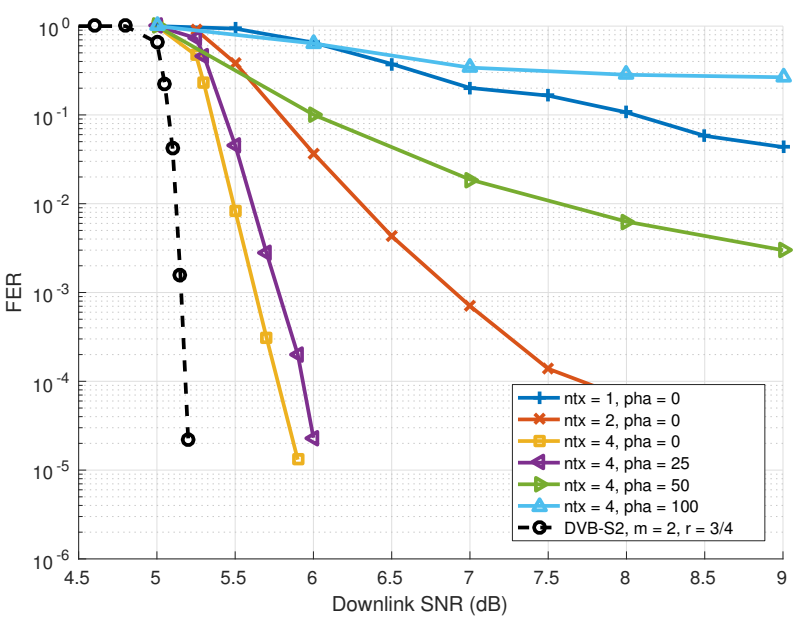

(b) $\sigma_{I}^{2}=0.4$

Figure 6. Micro diversity in analogue transparent systems with $P_{T}=2400 \mathrm{~W}$.

\section{Conclusion and future research}

In this paper we investigate the transmitter micro diversity technique for satellite systems with optical feeder link. Up to four independent optical beams are transmitted simultaneously in order to combat the strong fading. Perfect and imperfect knowledge of the phases of each beam is considered at the receiver. The transmit powers are respectively fixed to $50 \mathrm{~W}$ and $2400 \mathrm{~W}$ for DTS and ATS. A detailed discussion is presented to justify these chosen powers for each system scenario. Our simulation results indicate that with perfect knowledge of the phases and for low scintillation indexes, two beams are sufficient to reach the target FER of $10^{-5}$ with a loss of less than $1 \mathrm{~dB}$ in downlink SNR. For DTSs, the loss is actually negligible. On the other hand, for large scintillation indexes, four transmitted beams with at least some partial knowledge of the phases are needed in order to allow losses less than $1 \mathrm{~dB}$ at target FER of $10^{-5}$. In this paper we confine ourselves to the micro diversity techniques. It is interesting to also investigate the effect of the macro diversity, i.e., when several ground stations are available. A combination of both techniques may indeed provide a very promising system performance. Some research in this direction is on going.

\section{References}

1 "NetWorld2020s SatCom WG The role of satellites in 5G," WhitePaper Version 5, NetWorld2020, July 2014.

2 "Broadband Access via Integrated Terrestrial and Satellite Systems (BATS)," European Project, 2012.

${ }^{3}$ Muhammad, M., Giambene, G., and Cola, T. d., "Channel prediction and network coding for smart gateway diversity in terabit satellite networks," 2014 IEEE Global Communications Conference, Dec. 2014, pp. 3549-3554.

${ }^{4}$ Gharanjik, A., Rao, B., Arapoglou, P.-D., and Ottersten, B., "Large scale transmit diversity in Q/V band feeder link with multiple gateways," 2013 IEEE 24th International Symposium on Personal Indoor and Mobile Radio Communications (PIMRC), Sept. 2013, pp. 766-770.

${ }^{5}$ Kyrgiazos, A., Evans, B., and Thompson, P., "On the Gateway Diversity for High Throughput Broadband Satellite Systems," IEEE Transactions on Wireless Communications, Vol. 13, No. 10, Oct. 2014, pp. 5411-5426.

${ }^{6}$ Candreva, E. A., Suffritti, R., and Dervin, M., "Increasing the feeder link efficiency in broadband satellite systems," 2014 7 th Advanced Satellite Multimedia Systems Conference and the 13th Signal Processing for Space Communications Workshop (ASMS/SPSC), Sept. 2014, pp. 300-305.

7 "1st ESA W-Band SATCOM Workshop," WorkShop, ESA, ESA/ESTEC Noordwijk, The Netherlands, April 2015.

${ }^{8}$ Cowley, W., Giggenbach, D., and Calvo, R., "Optical transmission schemes for GEO feeder links," 2014 IEEE International Conference on Communications (ICC), June 2014, pp. 4154-4159.

${ }^{9}$ Gharanjik, A., Liolis, K., Shankar, M., and Ottersten, B., "Spatial multiplexing in optical feeder links for high throughput satellites," 2014 IEEE Global Conference on Signal and Information Processing (GlobalSIP), Dec. 2014, pp. $1112-1116$.

${ }^{10}$ Dimitrov, S., Matuz, B., Liva, G., Barrios, R., Mata-Calvo, R., and Giggenbach, D., "Digital modulation and coding for satellite optical feeder links," Advanced Satellite Multimedia Systems Conference and the 13th Signal Processing for Space Communications Workshop (ASMS/SPSC), 2014 7th, Sept. 2014, pp. 150-157.

${ }^{11}$ Ozbilgin, T. and Koca, M., "Optical Spatial Modulation Over Atmospheric Turbulence Channels," Journal of Lightwave Technology, Vol. 33, No. 11, June 2015, pp. 2313-2323. 
${ }^{12}$ Mesleh, R., Elgala, H., and Haas, H., "Optical Spatial Modulation," IEEE/OSA Journal of Optical Communications and Networking, Vol. 3, No. 3, March 2011, pp. 234-244.

${ }^{13}$ Safari, M. and Uysal, M., "Do We Really Need OSTBCs for Free-Space Optical Communication with Direct Detection?" IEEE Transactions on Wireless Communications, Vol. 7, No. 11, Nov. 2008, pp. 4445-4448.

${ }^{14}$ Yang, G., Khalighi, M.-A., Virieux, T., Bourennane, S., and Ghassemlooy, Z., "Contrasting space-time schemes for MIMO FSO systems with non-coherent modulation," 2012 International Workshop on Optical Wireless Communications (IWOW), Oct. 2012, pp. 1-3.

${ }^{15}$ Simon, M. K. and Vilnrotter, V., "Alamouti-type space-time coding for free-space optical communication with direct detection," IEEE Transactions on Wireless Communications, Vol. 4, No. 1, Jan. 2005, pp. 35-39.

${ }^{16}$ Park, J., Lee, E., and Yoon, G., "Average Bit-Error Rate of the Alamouti Scheme in Gamma-Gamma Fading Channels," IEEE Photonics Technology Letters, Vol. 23, No. 4, Feb. 2011, pp. 269-271.

${ }^{17}$ Song, X. and Cheng, J., "Alamouti-type STBC for subcarrier intensity modulated wireless optical communications," 2012 IEEE Global Communications Conference (GLOBECOM), Dec. 2012, pp. 2936-2940.

${ }^{18}$ Ren, T.-P., Yuen, C., Guan, Y. L., and Tang, G.-S., "High-Order Intensity Modulations for OSTBC in Free-Space Optical MIMO Communications," IEEE Wireless Communications Letters, Vol. 2, No. 6, Dec. 2013, pp. 607-610.

${ }^{19}$ Bhatnagar, M. and Anees, S., "On the Performance of Alamouti Scheme in Gamma-Gamma Fading FSO Links With Pointing Errors," IEEE Wireless Communications Letters, Vol. 4, No. 1, Feb. 2015, pp. 94-97.

${ }^{20}$ Barrios, R., Mata-Calvo, R., and Giggenbach, D., "RivOLi-TN1: Review of RoFSO techniques for Optical GEO Feeder Links," Jan. 2015.

21 "Second generation framing structure, channel coding and modulation systems for Broadcasting, Interactive Services, News Gathering and other broadband satellite applications; Part 1 (DVB-S2)," Tech. Rep. EN 302 307-1 V1.4.1, ETSI, Nov. 2014.

${ }^{22} \mathrm{Li}$, J., Liu, J. Q., and Taylor, D., "Optical Communication Using Subcarrier PSK Intensity Modulation Through Atmospheric Turbulence Channels," IEEE Transactions on Communications, Vol. 55, No. 8, Aug. 2007, pp. 1598-1606.

${ }^{23}$ Ghassemlooy, Z., Popoola, W., Gao, S., Allen, J., and Leitgeb, E., "Free-space optical communication employing subcarrier modulation and spatial diversity in atmospheric turbulence channel," IET Optoelectronics, Vol. 2, No. 1, Feb. 2008, pp. 16-23.

${ }^{24}$ Popoola, W. and Ghassemlooy, Z., "BPSK Subcarrier Intensity Modulated Free-Space Optical Communications in Atmospheric Turbulence," Journal of Lightwave Technology, Vol. 27, No. 8, April 2009, pp. 967-973.

${ }^{25}$ Samimi, H., "Optical Communication Using Subcarrier Intensity Modulation Through Generalized Turbulence Channels," Journal of Optical Communications and Networking, Vol. 4, No. 5, May 2012, pp. 378.

${ }^{26}$ Andrews, L. C. and Phillips, R. L., Laser Beam Propagation through Random Media, Vol. PM152, SPIE, 2005.

${ }^{27}$ Navidpour, S., Uysal, M., and Li, J., "BER performance of MIMO free-space optical links," Vehicular Technology Conference, 2004. VTC2004-Fall. 2004 IEEE 60th, Vol. 5, Sept. 2004, pp. 3378-3382 Vol. 5.

${ }^{28}$ Moradi, H., Falahpour, M., Refai, H. H., LoPresti, P. G., and Atiquzzaman, M., "BER analysis of optical wireless signals through lognormal fading channels with perfect CSI," IEEE, 2010, pp. 493-497.

${ }^{29}$ Yang, L., Cheng, J., and Holzman, J. F., "Optical communications over lognormal fading channels using OOK," IEEE, Oct. 2013, pp. 99-103.

${ }^{30}$ Ansari, I., Alouini, M.-S., and Cheng, J., "On the Capacity of FSO Links under Lognormal and Rician-Lognormal Turbulences," Vehicular Technology Conference (VTC Fall), 2014 IEEE 80th, Sept. 2014, pp. 1-6.

${ }^{31}$ Romba, J., Sodnik, Z., Reyes, M., Alonso, A., and Bird, A., "ESA's bidirectional space-to-ground laser communication experiments," Oct. 2004, p. 287.

${ }^{32}$ Dimitrov, S., Barrios, R., Matuz, B., Liva, G., Mata-Calvo, R., and Giggenbach, D., "Digital modulation and coding for satellite optical feeder links with pre-distortion adaptive optics," International Journal of Satellite Communications and Networking, Jan. 2015, pp. n/a-n/a.

${ }^{33}$ Iizuka, K., Elements of Photonics, Vol. 2, Wiley, 2002.

34 "DVB-S2 Implementation Guidelines," Tech. Rep. TR 102 376-1 V1.2.1, ETSI, Nov. 2015. 